\title{
Reducing Emergence: \\ The Case Studies in Statistic Thermodynamics, General Relativity, and Quantum Mechanics
}

\author{
Vasil Penchev, vasildinev@gmail.com \\ Bulgarian Academy of Sciences: Institute of Philosophy and Sociology: \\ Dept. of Logical Systems and Models
}

\begin{abstract}
The emergent properties are properties referring to a system as a whole, but they do not make sense to its elements or parts being small enough. Furthermore certain emergent properties are reducible to those of elements or relevant parts often. The paper means the special case where the description of the system by means of its emergent properties is much simpler than that of its relevant elements or parts. The concept is investigated by a case study based on statistic thermodynamics, general relativity, and quantum mechanics.
\end{abstract}

Key words: emergent property, reduction, statistic thermodynamics, general relativity, quantum mechanics

Reducing emergence is defined as an essential simplification of the description of a complex system in comparison with the descriptions of at least some parts of it. The emergent property has a "negative sign": It is expressed in simplifying the description of the system. A cause for this can be the very strong interactions between the parts, which restrict the degrees of freedom (DOF) of the system. Then DOF of the system can be essentially less than those of at least some parts, which can simplify enough its description.

Another option of reducing emergence is the statistic description of the system: It supposes that the system consists of a huge number of identical elements differing in a few quantities. The system can be exhaustedly described by the averages of their distributions in all elements. The huge variety in the individual values of those quantities is replaced by their integral averages as to the behavior of the system. One can offer hypothesis that the reducing emergence for any strong interaction between the parts of a system and that for its statistical description are equivalent to each other under certain conditions.

The model given by statistic thermodynamics describes the state of a system as a whole featured by quantities like volume, temperature, pressure, energy, entropy, etc. in terms of a statistical ensemble of elements like atoms or molecules, which are considered as moving material points. The distributions of variables of their individual movements like position, speed, acceleration, imply the thermodynamic quantities of the system like the enumerated above. The description of the statistical ensemble including descriptions of all elements is incredibly difficult, bulky, and practically impossible while the derivative one of the whole as a 
thermodynamic system is reduced to the initial values and their change in time of a few thermodynamic quantities. Though the system is much more complex, the thermodynamic description of its behavior as a whole is exceptionally simplified for statistical averaging in the huge number of its elements. The more elements are there, the description is simpler for the law of large numbers in statistics.

General relativity can be deduced (Jacobson 1995) from the laws of classical thermodynamics and the Bekenstein bound (Bekenstein 1973). The Bekenstein bound can be considered as an additional thermodynamic law about the upper limit of entropy, which is reached for electromagnetic radiation. So general relativity should be entirely a thermodynamic theory being deduced from a few thermodynamic laws. However the Bekenstein bound is a quantum law in the following sense: Two conjugates like energy and time (for the radius of a corresponding ball) are equated reversely proportionally. This implies the reverse proportionality of their differentials, which cannot be simultaneously given. Consequently general relativity should be accepted as a quantum thermodynamic theory. One can investigate its reduction to some corresponding statistical ensemble in the model given of statistic thermodynamics. That ensemble turns out to be all points of space-time differing from each other in the values of gravitational field featuring all points. This corresponds to returning the conception of "ether" in general relativity (Einstein 1920) after being driven out by special relativity as redundant.

Just because of the quantum essence of the Bekenstein bound, general relativity as a statistical thermodynamic theory shows an important difference in comparison with statistic thermodynamics: The whole of the considered isolated system (even the true universe) returns to all points of it in a way, which can be designated as cyclic and kinematic, i.e. per a unit of energy (mass) distributing the total energy of the system between all points of it according to the measures of their energy (mass) to the total one in the previous state. So the way of general relativity to be a statistic thermodynamic theory is inverted to that of statistic thermodynamics: It is a theory about the way for the whole to return to its statistical ensemble. Thus general relativity can contribute much to the conception of reducing emergence for this fundamental peculiarity: The statistic reducing emergence of that kind allows of the whole of the system to return to any element of it for the equal complexity (or the quantity of information) of the system after statistic reducing and that of the element. That reducing emergence should be designated as cyclic. Furthermore the cyclic identification of the system and any element of it can be extended so that all system to be considered as "inserted" in any element of it: This is the essence of "gauge ideology" in quantum mechanics and directs to it.

That cyclic reducing emergence interprets quantum mechanics as follows: Each quantity of any pair of conjugates can be seen as some statistic averaging and thus simplifying of the other being a functional of the distribution of the other. The same is embedded in the basic mathematical structure of quantum mechanics, that of the two dual and identical complex Hilbert spaces. It can be deduced from the fundamental postulate of quantum mechanics: It studies the system of quantum entities and macroscopic apparatuses by the indications of the latters. So an apparatus chooses randomly a value of the measured quantity among all possible ones. The theorem of the absence of hidden variables in quantum mechanics (Kochen, Specker 1967) 
excludes any well-ordering before measurement and thus needs the well-ordering theorem equivalent to the axiom of choice. The cyclic reducing emergence repeats this argument symmetrically for the other conjugate. Thus one can deduce the classical one-to-one mapping for any two conjugates in quantum mechanics if the unorderable coherent state featuring quantum mechanics is identified for both conjugates.

\section{References:}

Bekenstein, Jacob (1973) “Black Holes and Entropy,” Physical Review D 7 (8): 2333-2346.

Kochen, Simon and Ernst Specker (1967) "The problem of hidden variables in quantum mechanics," Physical Review A 17 (1): 59-87.

Jacobson, Ted (1995) "Thermodynamics of Spacetime: The Einstein Equation of State," Physical Review Letters 75 (7): 1260-1263

Einstein, Albert (1920) Äther und Relativitätstheorie, Berlin, Springer. 